\title{
Stochastic Restricted Estimation in Partially Linear Measurement Error Models
}

\author{
Chenyang Zhang ${ }^{1}$, Chuanhua $\mathrm{Wei}^{1}$ \& Bailing $\mathrm{An}^{2}$ \\ ${ }^{1}$ School of Science, Minzu University of China, Beijing, China \\ ${ }^{2}$ School of Mathematical Science, Huaibei Normal University, Huaibei, China \\ Correspondence: Chuan-hua Wei, Department of Statistics, School of Science, Minzu University of China,Beijing 100081, \\ China.
}

Received: February 26, 2018 Accepted: March 13, 2018 Online Published: April 18, 2018

doi:10.5539/ijsp.v7n3p66 URL: https://doi.org/10.5539/ijsp.v7n3p66

The research is financed by the Natural Science Foundation of Anhui Province, China(No.KJ2014B13).

\begin{abstract}
As a generalization of nonparametric regression model, partially linear model has been studied extensively in the last decades. This paper considers estimation of the semiparametric model under the situation that the covariates are measured with additive error in the linear part and some additional stochastic linear restrictions exist on the parametric component. Based on the corrected profile least-squares approach and mixed regression method, we propose a stochastic restricted estimator named the corrected profile mixed estimator for the parametric component, and discuss its statistical properties. We also construct a weighted stochastic restricted estimation for the parametric component. Finally, the proposed procedure is illustrated by simulation studies.
\end{abstract}

Keywords: partially linear models, measurement error, stochastic restricted estimation, corrected profile least-squares approach, mixed regression estimation

\section{Introduction}

In the last two decades, semiparametric measurement error (errors-in-variables) models have received considerable attention in statistics and econometrics. In this paper, the following structural partially linear errors-in-variables model is considered

$$
\left\{\begin{array}{l}
y_{i}=\mathbf{x}_{i}^{\mathrm{T}} \boldsymbol{\beta}+f\left(t_{i}\right)+\varepsilon_{i}, \quad i=1,2, \cdots, n, \\
\mathbf{v}_{i}=\mathbf{x}_{i}+\boldsymbol{\xi}_{i},
\end{array}\right.
$$

where $y_{i}$ are response, $\mathbf{x}_{i}=\left(x_{i 1}, x_{i 2}, \cdots, x_{i p}\right)^{\mathrm{T}}$ is a vector of random explanatory variables, $t_{i}$ is a scalar covariate, $\boldsymbol{\beta}=$ $\left(\beta_{1}, \beta_{2}, \cdots, \beta_{p}\right)^{\mathrm{T}}$ is a vector of $\mathrm{p}$-dimensional parameters which are unknown, $f(\cdot)$ is an unknown smooth function, and the residuals $\varepsilon_{i}$ 's are independent and identical distribution with zero mean and finite variance $\sigma^{2}$. The measurement errors $\boldsymbol{\xi}_{i}$ are independent and identically distributedwith mean zero and covariance matrix $\boldsymbol{\Sigma}_{\boldsymbol{\xi}} . \boldsymbol{\Sigma}_{\boldsymbol{\xi}}$ is assumed to be known. In the meantime, $\varepsilon_{i}$ 's are independent of $\left(\mathbf{x}_{i}^{\mathrm{T}}, t_{i}\right)$ 's and each $\boldsymbol{\xi}_{i}$ is independent of $\left(y_{i}, \mathbf{x}_{i}, t_{i}\right)$.

Obviously, model (1.1) is a generalization of the linear measurement error model and partially linear model, and has been discussed by many authors in the last two decades. To estimate $\beta$, Liang et al.(1999) proposed a corrected two-step method, Wang(1999) constructed an estimator using validation data. When the ratio of $\Sigma_{\xi}$ to $\sigma^{2}$ is known, Cui and Li (1998) proposed a profile total least-square estimator.

In practice, some outside sources can provide some prior information about the regression coefficients. Such information can be expressed as some exact or stochastic restrictions on the unknown regression parameters. Use of such restrictions on parameters may improve upon the efficiency of the estimator, and details can be found in Toutenburg(1982) and Rao et al. (2008).

In recent years, measurement error models with exact or stochastic restrictions have been studied by many authors. for the linear measurement error models, Shalabh et al. (2007) and Shalabh et al. (2009) studied the exact restricted estimation. Shalabh et al. (2010) and Li and Yang (2013) studied the estimation of the model when stochastic linear restrictions on regression coefficients are available. For the partially linear measurement error model with exact linear restrictions, Wei et al. (2013) proposed a restricted corrected profile least-squares estimator for the parametric component. In this paper, we consider the estimation of model (1.1) with the stochastic linear restrictions. The stochastic restrictions can be defined as

$$
\mathbf{b}=\mathbf{A} \beta+\eta,
$$


where $\mathbf{b}$ is a $k \times 1$ known vector and $\mathbf{A}$ is a $k \times p$ known matrix of full row rank. Further, the random error $\boldsymbol{\eta}$ is assumed to be distributed with mean vector $\mathbf{0}$ and covariance matrix $\sigma^{2} \boldsymbol{\Omega}$, where $\boldsymbol{\Omega}$ is a known positive definite matrix. Based on the corrected profile least-squares approach of Liang et al.(1999), and the mixed regression estimation method of Theil and Goldberger (1961) and Theil (1963), a stochastic restricted estimator named corrected profile mixed estimator for the parametric component $\boldsymbol{\beta}$ is defined. As a result, we extend, on one hand, the results of Shalabh et al. (2010) and Li and Yang (2013) from linear measurement error models to semiparametric measurement error models, and on the other hand, the results of Wei et al. (2013) from exact restrictions to the case of stochastic restrictions.

This paper is organized as follows. In Section 2, we construct the stochastic restricted estimators for the parametric component, and discuss their statistical properties. To examine the performance of the proposed approaches, we conduct some simulations in Section 3 . The proofs of main results are given in Section 4.

\section{Corrected Profile Mixed Estimation}

Let $y_{i}^{*}=y_{i}-\mathbf{x}_{i}^{\mathrm{T}} \boldsymbol{\beta}$, then we can get the following standard nonparametric regression model

$$
y_{i}^{*}=f\left(t_{i}\right)+\varepsilon_{i} \quad i=1,2, \cdots, n .
$$

We apply the local linear approach to estimate the function $f(\cdot)$. For a given point $t_{0}$, we have

$$
f(t) \approx f\left(t_{0}\right)+f^{\prime}\left(t_{0}\right)\left(t-t_{0}\right)
$$

for $t$ in a neighborhood of $t_{0}$ is available. Let $K(\cdot)$ be a kernel function and $h$ be a bandwidth, the local linear regression approach gives the solution of local parameters $\left\{f\left(t_{0}\right), f^{\prime}\left(t_{0}\right)\right\}$ by minimizing

$$
\sum_{i=1}^{n}\left[y_{i}^{*}-f\left(t_{0}\right)-f^{\prime}\left(t_{0}\right)\left(t_{i}-t_{0}\right)\right]^{2} K_{h}\left(t_{i}-t_{0}\right)
$$

where $K_{h}(\cdot)=K(\cdot / h) / h$. The solution to problem (2.2) is given by

$$
\left(\hat{f}\left(t_{0}\right), \hat{f}^{\prime}\left(t_{0}\right)\right)^{\mathrm{T}}=\left\{\mathbf{D}_{t_{0}}^{\mathrm{T}} \mathbf{W}_{t_{0}} \mathbf{D}_{t_{0}}\right\}^{-1} \mathbf{D}_{t_{0}}^{\mathrm{T}} \mathbf{W}_{t_{0}}(\mathbf{Y}-\mathbf{X} \boldsymbol{\beta})
$$

where $\mathbf{W}_{t_{0}}=\operatorname{diag}\left(K_{h}\left(t_{1}-t_{0}\right), K_{h}\left(t_{2}-t_{0}\right), \cdots, K_{h}\left(t_{n}-t_{0}\right)\right)$ and

$$
\mathbf{Y}=\left[\begin{array}{c}
y_{1} \\
y_{2} \\
\vdots \\
y_{n}
\end{array}\right], \mathbf{X}=\left[\begin{array}{c}
\mathbf{x}_{1}^{\mathrm{T}} \\
\mathbf{x}_{2}^{\mathrm{T}} \\
\vdots \\
\mathbf{x}_{n}^{\mathrm{T}}
\end{array}\right], \mathbf{D}_{\mathrm{t}_{0}}=\left[\begin{array}{cc}
1 & t_{1}-t_{0} \\
1 & t_{2}-t_{0} \\
\vdots & \vdots \\
1 & t_{n}-t_{0}
\end{array}\right] .
$$

Replacing $f\left(t_{i}\right)$ in $(2.1)$ with $\hat{f}\left(t_{i}\right)$, we can obtain the synthetic linear regression model after some algebraic operation

$$
y_{i}-\hat{y}_{i}=\left(\mathbf{x}_{i}-\hat{\mathbf{x}}_{i}\right)^{\mathrm{T}} \boldsymbol{\beta}+\tilde{\varepsilon}_{i},
$$

where $\tilde{\varepsilon}_{i}=\varepsilon_{i}+f\left(t_{i}\right)-\hat{f}\left(t_{i}\right), \hat{\mathbf{Y}}=\left(\hat{y}_{1}, \cdots, \hat{y}_{n}\right)^{\mathrm{T}}=\mathbf{S Y}, \hat{\mathbf{X}}=\left(\hat{\mathbf{x}}_{1}, \cdots, \hat{\mathbf{x}}_{n}\right)^{\mathrm{T}}=\mathbf{S X}$, with

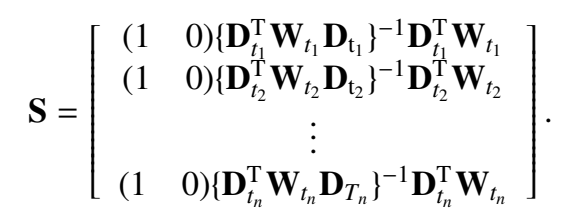

The profile least squares estimator for $\beta$ can be obtained with linear model (2.4). While $\mathbf{x}_{i}$ cannot be exactly observed in our case, the resulting estimator is inconsistent if we ignore the measurement error and replaces $\mathbf{x}_{i}$ by $\mathbf{v}_{i}$ in (2.4). By the correction for attenuation technique, the corrected profile least squares estimator of $\boldsymbol{\beta}$ can be defined as

$$
\hat{\boldsymbol{\beta}}=\arg \min _{\boldsymbol{\beta} \in R^{p}}\left[(\overline{\mathbf{Y}}-\overline{\mathbf{V}} \boldsymbol{\beta})^{\mathrm{T}}(\overline{\mathbf{Y}}-\overline{\mathbf{V}} \boldsymbol{\beta})-n \boldsymbol{\beta}^{\mathrm{T}} \boldsymbol{\Sigma}_{\xi} \boldsymbol{\beta}\right]=\left(\overline{\mathbf{V}}^{\mathrm{T}} \overline{\mathbf{V}}-n \boldsymbol{\Sigma}_{\xi}\right)^{-1} \overline{\mathbf{V}}^{\mathrm{T}} \overline{\mathbf{Y}},
$$

where $\overline{\mathbf{Y}}=\mathbf{Y}-\hat{\mathbf{Y}}, \overline{\mathbf{V}}=\mathbf{V}-\hat{\mathbf{V}}, \hat{\mathbf{V}}=\left(\hat{\mathbf{v}}_{1}, \cdots, \hat{\mathbf{v}}_{n}\right)^{\mathrm{T}}=\mathbf{S V}$ and $\mathbf{V}=\left(\mathbf{v}_{1}, \cdots, \mathbf{v}_{n}\right)^{\mathrm{T}}$.

According to Theil and Goldberger (1961), based on the stochastic prior information, the corrected profile least-squares mixed estimator can be given by minimizing the following cost function

$$
F(\boldsymbol{\beta})=(\overline{\mathbf{Y}}-\overline{\mathbf{V}} \boldsymbol{\beta})^{\mathrm{T}}(\overline{\mathbf{Y}}-\overline{\mathbf{V}} \boldsymbol{\beta})-n \boldsymbol{\beta}^{\mathrm{T}} \boldsymbol{\Sigma}_{\xi} \boldsymbol{\beta}+(\mathbf{b}-\mathbf{A} \boldsymbol{\beta})^{\mathrm{T}} \boldsymbol{\Omega}^{-1}(\mathbf{b}-\mathbf{A} \boldsymbol{\beta}),
$$


with respect to $\beta$.

By (2.7), we can obtain the following equation

$$
\frac{\partial F(\boldsymbol{\beta})}{\partial \boldsymbol{\beta}}=-2 \overline{\mathbf{V}}^{\mathrm{T}}(\overline{\mathbf{Y}}-\overline{\mathbf{V}} \boldsymbol{\beta})-2 n \boldsymbol{\Sigma}_{\xi} \boldsymbol{\beta}-2 \mathbf{A}^{\mathrm{T}} \boldsymbol{\Omega}^{-1}(\mathbf{b}-\mathbf{A} \boldsymbol{\beta})=\mathbf{0},
$$

Solving the equation (2.8), the corrected profile mixed estimator of $\beta$ is obtained as

$$
\hat{\boldsymbol{\beta}}_{\text {cpm }}=\left(\overline{\mathbf{V}}^{\mathrm{T}} \overline{\mathbf{V}}-n \boldsymbol{\Sigma}_{\xi}+\mathbf{A}^{\mathrm{T}} \boldsymbol{\Omega}^{-1} \mathbf{A}\right)^{-1}\left(\overline{\mathbf{V}}^{\mathrm{T}} \overline{\mathbf{Y}}+\mathbf{A}^{\mathrm{T}} \boldsymbol{\Omega}^{-1} \mathbf{b}\right),
$$

Denote $\mathbf{E}=\overline{\mathbf{V}}^{\mathrm{T}} \overline{\mathbf{V}}-n \boldsymbol{\Sigma}_{\boldsymbol{\xi}}$, by the Theorem A.18 of Rao et al (2008)(Page. 494), we have

$$
\left(\overline{\mathbf{V}}^{\mathrm{T}} \overline{\mathbf{V}}-n \boldsymbol{\Sigma}_{\xi}+\mathbf{A}^{\mathrm{T}} \mathbf{\Omega}^{-1} \mathbf{A}\right)^{-1}=\mathbf{E}^{-1}-\mathbf{E}^{-1} \mathbf{A}^{\mathrm{T}}\left[\mathbf{\Omega}+\mathbf{A} \mathbf{E}^{-1} \mathbf{A}^{\mathrm{T}}\right]^{-1} \mathbf{A} \mathbf{E}^{-1},
$$

Then, by (2.9) and (2.10), $\hat{\boldsymbol{\beta}}_{\mathrm{m}}$ can be rewritten as

$$
\hat{\boldsymbol{\beta}}_{\mathrm{cpm}}=\hat{\boldsymbol{\beta}}-\left(\overline{\mathbf{V}}^{\mathrm{T}} \overline{\mathbf{V}}-n \boldsymbol{\Sigma}_{\xi}\right)^{-1} \mathbf{A}^{\mathrm{T}}\left[\mathbf{\Omega}+\mathbf{A}\left(\overline{\mathbf{V}}^{\mathrm{T}} \overline{\mathbf{V}}-n \boldsymbol{\Sigma}_{\xi}\right)^{-1} \mathbf{A}^{\mathrm{T}}\right]^{-1}(\mathbf{A} \hat{\boldsymbol{\beta}}-\mathbf{b}),
$$

where $\hat{\beta}$ is the corrected profile least-squares estimator of $\beta$ in (2.6).

The asymptotic normality of $\hat{\boldsymbol{\beta}}_{\text {cpm }}$ can be proven by the following theorem .

Theorem 2.1 Under the conditions in the Section 5, the corrected profile mixed estimator of $\boldsymbol{\beta}$ is asymptotically normal, namely,

$$
\sqrt{n}\left(\hat{\boldsymbol{\beta}}_{\mathrm{cpm}}-\boldsymbol{\beta}\right) \stackrel{d}{\longrightarrow} N(\mathbf{0}, \boldsymbol{\Omega}),
$$

where $\boldsymbol{\Sigma}_{1}=E\left\{\left[\mathbf{X}_{1}-E\left(\mathbf{X}_{1} \mid t_{1}\right)\right]\left[\mathbf{X}_{1}-E\left(\mathbf{X}_{1} \mid t_{1}\right)\right]^{\mathrm{T}}\right\}, \boldsymbol{\Sigma}_{2}=\mathrm{E}\left\{\left[\mathbf{X}_{1}-E\left(\mathbf{X}_{1} \mid t_{1}\right)\left(\varepsilon_{1}-\boldsymbol{\xi}_{1}^{\mathrm{T}} \boldsymbol{\beta}\right)\right]^{\otimes 2}\right\}+E\left(\varepsilon_{1}^{2} \boldsymbol{\xi}_{1} \boldsymbol{\xi}_{1}^{\mathrm{T}}\right)+E\left\{\left[\left(\boldsymbol{\xi}_{1} \boldsymbol{\xi}_{1}^{\mathrm{T}}-\boldsymbol{\Sigma}_{\xi}\right) \boldsymbol{\beta}\right]^{\otimes 2}\right\}$, $\mathbf{\Omega}=\boldsymbol{\Sigma}_{1}^{-1} \boldsymbol{\Sigma}_{2} \boldsymbol{\Sigma}_{1}^{-1}$, and $A^{\otimes 2}$ means $A A^{\mathrm{T}}$.

The estimator $\hat{\boldsymbol{\beta}}_{\mathrm{cpm}}$ is defined under the assumption that both the sample information and the prior information which is expressed as stochastic restrictions are equally important and weight equally in the statistical procedure. In practice, this assumption may violate. Schaffrin and Toutenburg (1990) proposed the weighted mixed regression estimation to fix the problem. Li and Yang (2013) have studied the weighted stochastic restricted estimation for linear errors-in-variables models. In the following, we consider the weighted stochastic restricted estimation for model (1.1). Similar to Li and Yang (2013), the weighted corrected profile least-squares mixed estimator can be obtained by minimizing the following objective function

$$
F_{w}(\boldsymbol{\beta})=(\overline{\mathbf{Y}}-\overline{\mathbf{V}} \boldsymbol{\beta})^{\mathrm{T}}(\overline{\mathbf{Y}}-\overline{\mathbf{V}} \boldsymbol{\beta})-n \boldsymbol{\beta}^{\mathrm{T}} \boldsymbol{\Sigma}_{\xi} \boldsymbol{\beta}+\omega(\mathbf{b}-\mathbf{A} \boldsymbol{\beta})^{\mathrm{T}} \mathbf{\Omega}^{-1}(\mathbf{b}-\mathbf{A} \boldsymbol{\beta}),
$$

with respect to $\beta$, and $\omega$ is a non-stochastic and non-negative scalar weight ranging from 0 to 1 . By differentiating function $F_{w}(\boldsymbol{\beta})$ with respect to $\beta$, we have the following equation

$$
\frac{\partial F_{w}(\boldsymbol{\beta})}{\partial \boldsymbol{\beta}}=-2 \overline{\mathbf{V}}^{\mathrm{T}}(\overline{\mathbf{Y}}-\overline{\mathbf{V}} \boldsymbol{\beta})-2 n \boldsymbol{\Sigma}_{\xi} \boldsymbol{\beta}-2 \omega \mathbf{A}^{\mathrm{T}} \mathbf{\Omega}^{-1}(\mathbf{b}-\mathbf{A} \boldsymbol{\beta})=\mathbf{0},
$$

Solving the equation (2.13), the weighted corrected profile mixed estimator of $\beta$ is obtained as

$$
\hat{\boldsymbol{\beta}}_{\mathrm{wcpm}}=\left(\overline{\mathbf{V}}^{\mathrm{T}} \overline{\mathbf{V}}-n \boldsymbol{\Sigma}_{\xi}+\omega \mathbf{A}^{\mathrm{T}} \boldsymbol{\Omega}^{-1} \mathbf{A}\right)^{-1}\left(\overline{\mathbf{V}}^{\mathrm{T}} \overline{\mathbf{Y}}+\omega \mathbf{A}^{\mathrm{T}} \mathbf{\Omega}^{-1} \mathbf{b}\right),
$$

The following theorem gives the asymptotic normality of $\hat{\boldsymbol{\beta}}_{\mathrm{wcpm}}$.

Theorem 2.2 Under the conditions in the Section 5, the corrected profile mixed estimator of $\beta$ is asymptotically normal, namely,

$$
\sqrt{n}\left(\hat{\boldsymbol{\beta}}_{\mathrm{wcpm}}-\boldsymbol{\beta}\right) \stackrel{d}{\longrightarrow} N(\mathbf{0}, \boldsymbol{\Omega}) .
$$

Remark 2.1 Theorems 2.1 and 2.2 indicate that both the corrected profile mixed estimator $\hat{\boldsymbol{\beta}}_{\mathrm{cpm}}$ and the weighted corrected profile mixed estimator $\hat{\boldsymbol{\beta}}_{\mathrm{wcpm}}$ and the corrected profile least-squares estimator $\hat{\boldsymbol{\beta}}$ have the same asymptotic distributions. The results is consistent with the results of Shalabh et al. (2010) and Li and Yang (2013) on the linear ultrastructural measurement error model with stochastic linear restrictions. As sample size increases, the results suggest that the effect of stochastic restrictions on the properties of estimators vanishes. 


\section{Simulation Studies}

In this section, we conduct some simulations to examine the finite sample performance of the proposed estimation procedure. The data are generated from the following partially linear errors-in-variables model

$$
\left\{\begin{array}{l}
y_{i}=x_{1} \beta_{1}+x_{2 i} \beta_{2}+x_{3} \beta_{3}+x_{4 i} \beta_{4}+f\left(t_{i}\right)+\varepsilon_{i} \\
v_{k i}=x_{k i}+e_{k i}, i=1,2, \cdots, n, k=1,2,3,4
\end{array}\right.
$$

where $x_{1 i} \sim N(0,1), x_{2 i} \sim N(1,2), x_{3 i} \sim U(-2,2), x_{4 i} \sim U(-1,1), t_{i} \sim U(0,1) \alpha\left(u_{i}\right)=\sin \left(2 \pi t_{i}\right)$, measurement errors $\mathbf{e}_{i}=\left(e_{1 i}, e_{2 i}, e_{3 i}, e_{4 i}\right) \sim N\left(\mathbf{0}, 0.25 \mathbf{I}_{4}\right)$.

To figure out the effect of the distribution of the error on our results, we take the following 4 different distributions which are adjusted to have the common variance $\sigma^{2}=0.25$, (1) $\varepsilon_{i} \sim N\left(0,0.5^{2}\right),(2) \varepsilon_{i} \sim U(-\sqrt{3} / 2, \sqrt{3} / 2)$, (3) $\varepsilon_{i} \sim \frac{1}{8} \chi_{8}^{2}-1$, (4) $\varepsilon_{i} \sim \sqrt{3} / 4 t(8)$. Our simulation studies set the kernel function $K(x)=\frac{15}{16}\left(1-x^{2}\right)^{2} I_{|x| \leq 1}$ and bandwidth $h=n^{-1 / 5}$. The true parameters are taken as $\left(\beta_{1}, \beta_{2}, \beta_{3}, \beta_{4}\right)=(1,1,2,2)$, and $n=100,120,150$ were considered, and the stochastic linear restrictions are $\beta_{1}+\beta_{2}=2+\eta_{1}$ and $\beta_{3}-\beta_{4}=\eta_{2}$ with

$$
E \eta_{1}=E \eta_{2}=0, \operatorname{Var}\left(\eta_{1}\right)=0.04, \operatorname{Var}\left(\eta_{1}\right)=0.09, \operatorname{Cov}\left(\eta_{1}, \eta_{2}\right)=0.03,
$$

that is

$$
\mathbf{A}=\left[\begin{array}{cccc}
1 & 1 & 0 & 0 \\
0 & 0 & 1 & -1
\end{array}\right], \boldsymbol{\Omega}=\left[\begin{array}{cc}
0.04 & 0.03 \\
0.03 & 0.09
\end{array}\right]
$$

The simulation applys the mean squared error (MSE) criterion to compare the performance of the estimators. For each setting, the simulation replicates 1000 . In each replication, the corrected profile least-squares estimator $\hat{\boldsymbol{\beta}}$, and the corrected profile mixed estimator $\hat{\boldsymbol{\beta}}_{\mathrm{cpm}}$ can be obtained. The estimated mean squared error (EMSE) for both $\hat{\boldsymbol{\beta}}$ and $\hat{\boldsymbol{\beta}}_{\mathrm{cpm}}$ is given, and more details are presented in Table 3.1. The EMSEs for the different estimators are calculated by:

$$
\operatorname{EMSE}\left(\boldsymbol{\beta}^{*}\right)=\frac{1}{1000} \sum_{j=1}^{500} \sum_{k=1}^{4}\left(\beta_{k j}^{*}-\beta_{k}\right)^{2},
$$

where $\beta_{k j}^{*}$ denotes the estimate of the $k$ th parameter in $j$ th replication and $\beta_{k}, k=1,2,3,4$ are the true parameter values above.

The simulation results can be summarized as follows. The EMSE of all the estimators decrease, as the sample size increases. In all these cases, the corrected profile mixed estimators show better performance than the corrected profile least-squares estimators.

Table 1. EMSEs of the estimators

\begin{tabular}{lrlrlrlrl}
\hline & $\varepsilon_{i} \sim$ & $N(0,0.25)$ & $\varepsilon_{i} \sim$ & $U\left(-\frac{\sqrt{3}}{2}, \frac{\sqrt{3}}{2}\right)$ & $\varepsilon_{i} \sim$ & $\frac{1}{8} \chi_{8}^{2}-1$ & $\varepsilon_{i} \sim$ & $\frac{\sqrt{3}}{4} t(8)$ \\
$n$ & $\hat{\boldsymbol{\beta}}$ & $\hat{\boldsymbol{\beta}}_{\mathrm{cpm}}$ & $\hat{\boldsymbol{\beta}}$ & $\hat{\boldsymbol{\beta}}_{\mathrm{cpm}}$ & $\hat{\boldsymbol{\beta}}$ & $\hat{\boldsymbol{\beta}}_{\mathrm{cpm}}$ & $\hat{\boldsymbol{\beta}}$ & $\hat{\boldsymbol{\beta}}_{\mathrm{cpm}}$ \\
\hline 100 & 0.830 & 0.118 & 0.871 & 0.119 & 0.837 & 0.121 & 0.848 & 0.123 \\
120 & 0.515 & 0.101 & 0.546 & 0.102 & 0.535 & 0.100 & 0.553 & 0.104 \\
150 & 0.348 & 0.090 & 0.345 & 0.084 & 0.307 & 0.083 & 0.323 & 0.086 \\
\hline
\end{tabular}

\section{Proof of the Main Results}

To derive the main results, the following assumptions are required. They are also assumed in Wei et al. (2013). These mild assumptions can be easily satisfied in most cases.

Assumption 1 The random variable thas a bounded support $\Psi$. Its density function $g(\cdot)$ is Lipschitz continuous and bounded away from 0 on its support.

Assumption 2 There is an $s>2$ such that $E\|\mathbf{X}\|^{2 s}<\infty$ and $E\|\boldsymbol{\xi}\|^{2 s}<\infty$ and for some $k<2-s^{-1}$ such that $n^{2 k-1} h \rightarrow \infty$.

Assumption $3 f(\cdot)$ have continuous second derivative in $t \in \boldsymbol{\Psi}$.

Assumption 4 The function $K(\cdot)$ is a symmetric density function with compact support and the bandwidth $h$ satisfies $n h^{8} \rightarrow 0$ and $n h^{2} /(\log n)^{2} \rightarrow \infty$. 
Proof of Theorem 2.1. Let $\boldsymbol{\Delta}=\overline{\mathbf{V}}^{\mathrm{T}} \overline{\mathbf{V}}-n \boldsymbol{\Sigma}_{\xi}+\mathbf{A}^{\mathrm{T}} \mathbf{\Omega}^{-1} \mathbf{A}$ and $\mathbf{U}=\left(\boldsymbol{\xi}_{1}, \cdots, \boldsymbol{\xi}_{n}\right)^{\mathrm{T}}, \mathbf{F}=\left[f\left(t_{1}\right), f\left(t_{2}\right), \cdots, f\left(t_{n}\right)\right]^{\mathrm{T}}$, by the definition of $\hat{\boldsymbol{\beta}}_{\mathrm{cpm}}$, we have

$$
\hat{\boldsymbol{\beta}}_{\mathrm{cpm}}=\boldsymbol{\beta}+\boldsymbol{\Delta}^{-1} \overline{\mathbf{X}}^{\mathrm{T}}\left(\mathbf{I}_{n}-\mathbf{S}\right) \mathbf{F}+\boldsymbol{\Delta}^{-1} \overline{\mathbf{V}}^{\mathrm{T}}\left(\mathbf{I}_{n}-\mathbf{S}\right)(\boldsymbol{\varepsilon}-\mathbf{U} \boldsymbol{\beta})+n \boldsymbol{\Sigma}_{\xi} \boldsymbol{\beta}+\boldsymbol{\Delta}^{-1} \mathbf{A}^{\mathrm{T}} \boldsymbol{\Omega}^{-1} \boldsymbol{\eta}
$$

By the Lemma 5.1 of Wei et al. (2013), let $\tilde{\mathbf{X}}_{i}=\mathbf{X}_{i}-E\left(\mathbf{X}_{i} \mid T_{i}\right)$, we have

$$
\begin{gathered}
\frac{1}{n}\left(\overline{\mathbf{V}}^{\mathrm{T}} \overline{\mathbf{V}}-n \boldsymbol{\Sigma}_{\xi}\right) \stackrel{p}{\longrightarrow} \boldsymbol{\Sigma}_{1} . \\
\frac{1}{n} \overline{\mathbf{V}}^{\mathrm{T}}\left(\mathbf{I}_{n}-\mathbf{S}\right) \mathbf{F}=o_{p}\left(n^{-1 / 2}\right), \\
\frac{1}{n} \overline{\mathbf{V}}^{\mathrm{T}}\left(\mathbf{I}-\mathbf{S}_{n}\right)(\boldsymbol{\varepsilon}-\mathbf{U} \boldsymbol{\beta})=\frac{1}{n} \sum_{i=1}^{n}\left(\tilde{\mathbf{X}}_{i}+\boldsymbol{\xi}_{i}\right)\left(\varepsilon_{i}-\boldsymbol{\xi}_{i}^{\mathrm{T}} \boldsymbol{\beta}\right)+o_{p}\left(n^{-1 / 2}\right) .
\end{gathered}
$$

We can obtain

$$
\frac{1}{\sqrt{n}} \mathbf{A}^{\mathrm{T}} \mathbf{\Omega}^{-1} \boldsymbol{\eta}=o_{p}(1)
$$

by the fact that

$$
\mathrm{E} \mathbf{A}^{\mathrm{T}} \mathbf{\Omega}^{-1} \boldsymbol{\eta}=\mathbf{0}, \quad \operatorname{Cov}\left(\mathbf{A}^{\mathrm{T}} \mathbf{\Omega}^{-1} \boldsymbol{\eta}\right)=\mathbf{A}^{\mathrm{T}} \boldsymbol{\Omega}^{-1} \mathbf{A}
$$

Therefore, combining the fact that $\frac{1}{n} \mathbf{A}^{\mathrm{T}} \mathbf{\Omega}^{-1} \mathbf{A}=o_{p}(1)$, it holds that

$$
\sqrt{n}\left(\hat{\boldsymbol{\beta}}_{\mathrm{cpm}}-\boldsymbol{\beta}\right)=\boldsymbol{\Sigma}_{1}^{-1} \frac{1}{\sqrt{n}} \sum_{i=1}^{n}\left[\left(\tilde{\mathbf{X}}_{i}+\boldsymbol{\xi}_{i}\right)\left(\varepsilon_{i}-\boldsymbol{\xi}_{i}^{\mathrm{T}} \boldsymbol{\beta}\right)+\boldsymbol{\Sigma}_{\xi} \boldsymbol{\beta}\right]+o_{p}(1) .
$$

Denote $\boldsymbol{\zeta}_{i}=\left(\tilde{\mathbf{X}}_{i}+\boldsymbol{\xi}_{i}\right)\left(\varepsilon_{i}-\boldsymbol{\xi}_{i}^{\mathrm{T}} \boldsymbol{\beta}\right)+\boldsymbol{\Sigma}_{\xi} \boldsymbol{\beta}$. Then for the i.i.d random vectors $\boldsymbol{\zeta}_{i}$, we have $E\left(\boldsymbol{\zeta}_{i}\right)=\mathbf{0}$ and $\operatorname{Cov}\left(\boldsymbol{\zeta}_{i}\right)=\boldsymbol{\Sigma}_{2}$. Then, by the multivariate central theorem, we have

$$
\frac{1}{\sqrt{n}} \sum_{i=1}^{n} \zeta_{i} \stackrel{D}{\longrightarrow} N\left(\mathbf{0}, \boldsymbol{\Sigma}_{2}\right)
$$

Then, combining (4.6) and (4.7), by the Slutsky theorem, we have

$$
\sqrt{n}\left(\hat{\boldsymbol{\beta}}_{\mathrm{cpm}}-\boldsymbol{\beta}\right) \stackrel{D}{\longrightarrow} N\left(\mathbf{0}, \boldsymbol{\Sigma}_{1}^{-1} \boldsymbol{\Sigma}_{2} \boldsymbol{\Sigma}_{1}^{-1}\right) .
$$

Proof of Theorem 2.2. The proof of Theorem 2.2 is similar to the proof of Theorem 2.1, we omit the details here.

\section{References}

Cui, H. J., \& Li, R. C. (1998). On parameter estimation for semi-linear errors-in-variables models. Journal of Multivariate Analysis, 64, 1-24. https://doi.org/10.1006/jmva.1997.1712

Li, W. X., \& Yang, H. (2013). Weighted Stochastic Restricted Estimation in Linear Measurement Error Models, Communications in Statistics - Simulation and Computation, 42(4), 932-968. https://doi.org/10.1080/03610918.2011.650260

Liang, H., \& Li, R. Z. (2009). Variable Selection for Partially Linear Models With Measurement Errors. Journal of the American Statistical Association, 104, 234-248. https://doi.org/10.1198/jasa.2009.0127

Liang, H., Hardle, W., \& Carroll, R. J. (1999). Estimation in a semiparametric partially linear errors-in-variables model. The Annals of Statistics, 27, 1519-1935.

Rao, C. R., Toutenburg, H., Shalabh, \& Heumann, C. (2008). Linear Models: Least Squares and Alternatives, Springer, Berlin.

Shalabh, Garg, G., \& Misra, N. (2010). Consistent estimation of regression coefficients in ultrastructural measurement error model using stochastic prior information. Statistical Papers, 51, 717-748. https://doi.org/10.1007/s00362-008$0162-\mathrm{z}$

Shalabh, Garg, G., \& Misra, N. (2009). Use of Prior Information in the Consistent Estimation of Regression Coefficients in a Measurement Error Model. Journal of Multivariate Analysis, 100, 1498-1520. https://doi.org/10.1016/j.jmva.2008.12.014 
Shalabh, Garg, G., \& Misra, N. (2007). Restricted regression estimation in measurement error models. Computational Statistics and Data Analysis', 52, 1149-1166. https://doi.org/10.1016/j.csda.2007.05.011

Theil, H. (1963). On the use of incomplete prior information in regression analysis. Journal of the American Statistical Association, 58, 401-414. https://doi.org/10.1080/01621459.1963.10500854

Theil, H., \& Goldberger, A. S. (1961). On pure and mixed statistical estimation in economics. International Economic Review, 2, 65-78. https://doi.org/10.2307/2525589

Toutenburg, H. (1982). Prior Information in Linear Models. Wiley, New York.

Wang, Q. H. (1999). Estimation of partial linear error-in-variables models with validation data. Journal of Multivariate Analysis, 69, 30-64. https://doi.org/10.1006/jmva.1998.1797

Wei, C. H., Jia, X. J., \& Hu, H. S. (2013). Restricted estimation in partially linear errors-in-variables models. Communications in Statistics-Simulation and Computation, 42(8), 1836-1847. https://doi.org/10.1080/03610918.2012.679759

You, J. H., \& Chen, G. M. (2006). Estimation of a semiparametric varying-coefficient partially linear errors-in-variables model. Journal of Multivariate Analysis, 97, 324-341. https://doi.org/10.1016/j.jmva.2005.03.002

\section{Copyrights}

Copyright for this article is retained by the author(s), with first publication rights granted to the journal.

This is an open-access article distributed under the terms and conditions of the Creative Commons Attribution license (http://creativecommons.org/licenses/by/4.0/). 